\title{
Capacity Optimizing Power Loading Scheme for Spatially Constrained Antenna Arrays: Channels with Narrow Angular Spread
}

\author{
Tharaka A. Lamahewa and Thushara D. Abhayapala \\ ANU College of Engineering and Computer Science \\ The Australian National University \\ Canberra ACT 0200, Australia \\ \{tharaka.lamahewa, thushara.abhayapala\}@anu.edu.au
}

\author{
Tony S. Pollock \\ National ICT Australia \\ Locked Bag 8001, \\ Canberra ACT 2601, Australia \\ tony.pollock@ieee.org
}

\begin{abstract}
This paper proposes a capacity optimizing power loading scheme for spatially constrained antenna arrays. The proposed power loading scheme is designed mainly for MIMO systems that are operating over channels with narrow angular spread values. The design problem is approached from a physical wave field perspective, in particular using a modal expansion for free space wave propagation. When a signal is transmitted from a spatially constrained antenna array, there will be an infinite number of modes excited at the transmit antenna region and only a finite number of those excited modes will have sufficient power to carry information to the other end of the channel. We show that in a non-isotropic scattering environment, correlation between adjacent modes is significantly higher than the correlation between well separated mode orders. Based on this finding, the power loading scheme is designed to eliminate the correlation between adjacent modes by allocating zero power to every second effective mode at the transmitter region. The capacity performance of the proposed scheme is evaluated for 1-D and 2-D antenna array geometries and results are compared with the traditional equal power loading scheme. We show that the proposed scheme gives more scope for capacity improvements for 1-D arrays at low angular spread values than for 2-D arrays.
\end{abstract}

\section{INTRODUCTION}

Multiple-input multiple-output (MIMO) communications systems using multi-antenna arrays simultaneously during transmission and reception have generated significant interest in recent years. Theoretical work of [1] showed the potential for significant capacity increases in wireless channels via spatial multiplexing with sparse antenna arrays. However, in reality, the capacity is significantly reduced when the antennas are insufficiently spaced (spatially constrained antenna arrays) or the scattering environment is non-isotropic [2-5], both resulting correlated channel gains. To improve the capacity in correlated fading environments, [1, 6-9] have proposed various power allocation schemes (or water filling strategies) assuming perfect CSI or partial CSI (e.g. channel covariance) is available at the transmitter through feedback (instantaneous/delayed). However, performance of these schemes heavily depends on the accuracy of the feedback information and the feedback rate.

In contrast, in this paper we derive a fixed power loading scheme which optimizes the capacity performance of spatially constrained antenna arrays operating over channels with narrow angular spread. The design of the power loading scheme is based on the fixed and known parameters of MIMO channels, namely the antenna spacing and antenna placement which are known at the transmitter. Therefore, with this design, the power loading scheme is fixed for fixed antenna placement and the transmitter does not require any form of CSI feedback from the receiver.

In this paper, we approach the power loading design problem from a physical wave field perspective. In particular using a 2-dimensional modal expansion for free space wave propagation, introduced in [10] for wireless channel modelling. When a signal is transmitted from a spatially constrained antenna array, there will be an infinite number of modes excited at the transmit antenna region and only a finite number of modes will have sufficient power to carry information to the other end of the channel. First we show that in a non-isotropic scattering environment, correlation between adjacent modes is significantly higher than the correlation between well separated mode orders. Motivated by this finding, we design the power loading scheme to eliminate the correlation between adjacent modes by allocating zero power to every second effective mode at the transmitter region.

Notations: Throughout the paper, the following notations will be used: Bold lower (upper) letters denote vectors (matrices). $[\cdot]^{T},[\cdot]^{*}$ and $[\cdot]^{\dagger}$ denote the transpose, complex conjugate and conjugate transpose operations, respectively. The symbols $\delta(\cdot)$ and $\otimes$ denote the Dirac delta function and Matrix Kronecker product, respectively. The notation $E\{\cdot\}$ denotes the mathematical expectation.

\section{CAPACITY OF SPATIALLY CONSTRAINED ANTENNA ARRAYS}

Consider a narrowband MIMO system consisting of $n_{T}$ transmit and $n_{R}$ receive antennas. In particular we consider the case where the receiver array consists of large number of 
receive antennas. It was shown in [11] that the total received power at the receiver array should remain a constant for a given region, regardless of the number of antennas in it. In this situation, the normalized ergodic capacity is given by

$$
\widetilde{C}=E\left\{\log \left|\boldsymbol{I}_{n_{R}}+\frac{1}{n_{R}} \boldsymbol{H} \boldsymbol{Q} \boldsymbol{H}^{\dagger}\right|\right\},
$$

where $\boldsymbol{H}$ is the $n_{R} \times n_{T}$ random flat fading channel matrix, $\boldsymbol{Q}=E\left\{\boldsymbol{x} \boldsymbol{x}^{\dagger}\right\}$ is the input signal covariance matrix and the scaling factor $1 / n_{R}$ scales the channel variances to $E\left\{\left|h_{r, t}\right|^{2}\right\} / n_{R}$, which assures the total received power remains a constant as the number of antennas is increased. In the following we will assume that the MIMO channel matrix $\boldsymbol{H}$ is fully known at the receiver and it is also partially known at the transmitter, where deterministic parts of the channel such as antenna spacing and antenna geometry are considered as partial channel information. In this work we utilize the 2-D spatial channel model derived in [10] to represent $\boldsymbol{H}$, which is described in the next sub-section.

\section{A. Spatial Channel Model}

Suppose $n_{T}$ transmit antennas located at positions $\boldsymbol{u}_{t}, t=$ $1,2, \cdots, n_{T}$ relative to the transmitter array origin, and $n_{R}$ receive antennas located at positions $\boldsymbol{v}_{r}, r=1,2, \cdots, n_{R}$ relative to the receiver array origin. $r_{T} \geq \max \left\|\boldsymbol{u}_{t}\right\|$ and $r_{R} \geq$ $\max \left\|\boldsymbol{v}_{r}\right\|$ denote the radius of spheres that contain all the transmitter and receiver antennas, respectively. We assume that scatterers are distributed in the far field from the transmitter and receiver antennas and regions containing the transmit and receive antennas are distinct.

Here we consider the situation where the multipath is restricted to the azimuth plane only (2-D scattering environment ${ }^{1}$ ), having no field components arriving at significant elevations. In this case, the channel matrix $\boldsymbol{H}$ can be decomposed as [10]

$$
\boldsymbol{H}=\boldsymbol{J}_{R} \boldsymbol{H}_{S} \boldsymbol{J}_{T}^{\dagger},
$$

where $\boldsymbol{J}_{R}$ is the $n_{R} \times\left(2 N_{R}+1\right)$ receiver configuration matrix,

$$
\boldsymbol{J}_{R}=\left[\begin{array}{ccc}
\mathcal{J}_{-N_{R}}\left(\boldsymbol{v}_{1}\right) & \cdots & \mathcal{J}_{N_{R}}\left(\boldsymbol{v}_{1}\right) \\
\mathcal{J}_{-N_{R}}\left(\boldsymbol{v}_{2}\right) & \cdots & \mathcal{J}_{N_{R}}\left(\boldsymbol{v}_{2}\right) \\
\vdots & \ddots & \vdots \\
\mathcal{J}_{-N_{R}}\left(\boldsymbol{v}_{n_{R}}\right) & \cdots & \mathcal{J}_{N_{R}}\left(\boldsymbol{v}_{n_{R}}\right)
\end{array}\right]
$$

$\boldsymbol{J}_{T}$ is the $n_{T} \times\left(2 N_{T}+1\right)$ transmitter configuration matrix,

$$
\boldsymbol{J}_{T}=\left[\begin{array}{ccc}
\mathcal{J}_{-N_{T}}\left(\boldsymbol{u}_{1}\right) & \cdots & \mathcal{J}_{N_{T}}\left(\boldsymbol{u}_{1}\right) \\
\mathcal{J}_{-N_{T}}\left(\boldsymbol{u}_{2}\right) & \cdots & \mathcal{J}_{N_{T}}\left(\boldsymbol{u}_{2}\right) \\
\vdots & \ddots & \vdots \\
\mathcal{J}_{-N_{T}}\left(\boldsymbol{u}_{n_{T}}\right) & \cdots & \mathcal{J}_{N_{T}}\left(\boldsymbol{u}_{n_{T}}\right)
\end{array}\right]
$$

with

$$
\mathcal{J}_{n}(\boldsymbol{x}) \triangleq J_{n}(k\|\boldsymbol{x}\|) e^{i n\left(\phi_{x}-\pi / 2\right)}
$$

as the spatial-to-mode function which maps the antenna location $\boldsymbol{x} \equiv\left(\|\boldsymbol{x}\|, \phi_{x}\right)$ in the polar coordinate system to the

\footnotetext{
${ }^{1}$ Similar results can be obtained using the 3 -D spatial model derived in [12]
}

$n$-th communication mode ${ }^{2}$ of the region, where $J_{n}(\cdot)$ are the Bessel functions of the first kind of order $n$ and $k=2 \pi / \lambda$ is the wave number with $\lambda$ the wave length. $2 N_{T}+1$ and $2 N_{R}+1$ are the number of effective ${ }^{3}$ communication modes at the transmit and receive regions, respectively. Note, $N_{T}$ and $N_{R}$ are defined by the size of the regions containing all the transmit and receive antennas, respectively [13]. In our case,

$$
N_{T}=\left\lceil\frac{k e r_{T}}{\lambda}\right\rceil \text { and } N_{R}=\left\lceil\frac{k e r_{R}}{\lambda}\right\rceil \text {, }
$$

where $e \approx 2.7183$.

Finally, $\boldsymbol{H}_{S}$ is the $\left(2 N_{R}+1\right) \times\left(2 N_{T}+1\right)$ random complex scattering channel matrix with $(p, q)$-th element given by

$$
\left\{\boldsymbol{H}_{S}\right\}_{p, q}=\int_{0}^{2 \pi} \int_{0}^{2 \pi} g(\phi, \psi) e^{i\left(q-N_{T}-1\right) \phi} e^{-i\left(p-N_{R}-1\right) \psi} \mathrm{d} \phi \mathrm{d} \psi
$$

representing the complex scattering gain between the $(q-$ $\left.N_{T}-1\right)$-th mode of the scatter-free transmit region and $\left(p-N_{R}-1\right)$-th mode of the scatter-free receiver region, where $g(\phi, \psi)$ is the effective random complex scattering gain function for signals with angle-of-departure $\phi$ from the scatterfree transmitter region and angle-of-arrival $\psi$ at the scatter-free receiver region.

The channel matrix decomposition (2) separates the channel into three distinct regions of interest: the scatter-free region around the transmitter antenna array, the scatter-free region around the receiver antenna array and the complex random scattering environment which is the complement of the union of two antenna array regions. In other words, MIMO channel is decomposed into deterministic and random matrices, where deterministic portions $\boldsymbol{J}_{T}$ and $\boldsymbol{J}_{R}$ represent the physical configuration of the transmitter and the receiver antenna arrays, respectively, and the random portion represents the complex scattering environment between the transmitter and the receiver antenna regions.

The rank of the channel matrix $\boldsymbol{H}$ gives the effective number of independent parallel channels between the transmit and receive antenna arrays, and thus determines the capacity of the communications system. From the decomposition (2), $\operatorname{rank}\{\boldsymbol{H}\}=\min \left\{\operatorname{rank}\left(\boldsymbol{J}_{T}\right), \operatorname{rank}\left(\boldsymbol{J}_{R}\right), \operatorname{rank}\left(\boldsymbol{H}_{s}\right)\right\}=$ $\min \left\{n_{R}, n_{T}, 2 N_{R}+1,2 N_{T}+1\right\}$. Therefore, if $2 N_{R}+1<n_{R}$ or $2 N_{T}+1<n_{T}$, the number of effective communication modes available at the transmit and receive regions limits the capacity of the system.

\section{Problem SETUP}

Substitution of (2) into (1) gives the ergodic capacity

$$
\widetilde{C}=E\left\{\log \left|\boldsymbol{I}_{n_{T}}+\frac{1}{n_{R}} \boldsymbol{Q} \boldsymbol{J}_{T} \boldsymbol{H}_{s}^{\dagger} \boldsymbol{J}_{R}^{\dagger} \boldsymbol{J}_{R} \boldsymbol{H}_{s} \boldsymbol{J}_{T}^{\dagger}\right|\right\} .
$$

${ }^{2}$ The set of modes form a basis of functions for representing a multipath wave field.

${ }^{3}$ Although there are infinite number of modes excited by an antenna array, there are only a finite number of modes $(2 \mathrm{~N}+1)$ which have sufficient power to carry information. 
Let $\widetilde{\boldsymbol{H}}=\boldsymbol{J}_{R} \boldsymbol{H}_{s}=\left[\widetilde{\boldsymbol{h}}_{1}^{\dagger}, \widetilde{\boldsymbol{h}}_{2}^{\dagger}, \cdots, \widetilde{\boldsymbol{h}}_{n_{R}}^{\dagger}\right]^{\dagger}$, where $\widetilde{\boldsymbol{h}}_{r}$ is a $1 \times\left(2 N_{T}+1\right)$ row-vector of $\widetilde{\boldsymbol{H}}$, which corresponds to the complex channel gains from $\left(2 N_{T}+1\right)$ transmit modes to the $r$-th receiver antenna, then the $\left(2 N_{T}+1\right) \times\left(2 N_{T}+1\right)$ transmitter modal correlation matrix can be defined as

$$
\boldsymbol{R}_{\widetilde{\boldsymbol{H}}} \triangleq E\left\{\widetilde{\boldsymbol{h}}_{r}^{\dagger} \widetilde{\boldsymbol{h}}_{r}\right\}, \forall r
$$

where $\left(n, n^{\prime}\right)$-th element of $\boldsymbol{R}_{\widetilde{\boldsymbol{H}}}$ gives the modal correlation between $n$-th and $n^{\prime}$-th modes in the transmit region. Similar to [14], we consider the situation where the receiver aperture of radius $r_{R}$ has optimally placed (uncorrelated) $n_{R}=2 N_{R}+1$ antennas, which corresponds to independent $\widetilde{\boldsymbol{h}}_{r}$ vectors, then the sample transmitter modal correlation matrix is given by

$$
\widehat{\boldsymbol{R}}_{\widetilde{\boldsymbol{H}}}=\frac{1}{n_{R}} \sum_{r=1}^{n_{R}} \widetilde{\boldsymbol{h}}_{r}^{\dagger} \widetilde{\boldsymbol{h}}_{r} .
$$

For a large number of receive antennas, the sample transmitter modal correlation matrix $\widehat{\boldsymbol{R}}_{\widetilde{\boldsymbol{H}}}$ converges to $\boldsymbol{R}_{\widetilde{\boldsymbol{H}}}$ as $r_{R} \rightarrow \infty$. Since $\widetilde{\boldsymbol{H}}^{\dagger} \widetilde{\boldsymbol{H}}=\sum_{r=1}^{n_{R}} \widetilde{\boldsymbol{h}}_{r}^{\dagger} \widetilde{\boldsymbol{h}}_{r}$, then for a large number of uncorrelated receive antennas, the ergodic capacity (5) converges to the deterministic quantity $C$,

$$
\begin{aligned}
\lim _{r_{R} \rightarrow \infty} \widetilde{C}=C \triangleq & \log \left|\boldsymbol{I}_{n_{T}}+\boldsymbol{Q} \boldsymbol{J}_{T} \boldsymbol{R}_{\widetilde{\boldsymbol{H}}} \boldsymbol{J}_{T}^{\dagger}\right|, \\
& \leq \log \left|\boldsymbol{I}_{n_{T}}+\boldsymbol{Q} \boldsymbol{J}_{T} \boldsymbol{J}_{T}^{\dagger}\right| .
\end{aligned}
$$

If the channel matrix $\boldsymbol{H}$ is known only to the receiver, then as shown in [1], transmission of statistically independent equal power signals each with a Gaussian distribution will be optimal. In this case $\boldsymbol{Q}=\left(P_{T} / n_{T}\right) \boldsymbol{I}_{n_{T}}$. In what follows we will refer to this scheme as equal power loading.

\section{A. Motivation}

First we derive the transmitter modal correlation matrix $\boldsymbol{R}_{\widetilde{H}}$ for a general scattering environment. The $1 \times\left(2 N_{T}+1\right)$ rowvector $\widetilde{\boldsymbol{h}}_{r}$ takes the form

$$
\begin{gathered}
\widetilde{\boldsymbol{h}}_{r}=\left[\sum_{n=-N_{R}}^{N_{R}} \mathcal{J}_{n}\left(\boldsymbol{v}_{r}\right) s_{n,-N_{T}}, \cdots, \sum_{n=-N_{R}}^{N_{R}} \mathcal{J}_{n}\left(\boldsymbol{v}_{r}\right) s_{n, m}, \cdots,\right. \\
\left.\cdots, \sum_{n=-N_{R}}^{N_{R}} \mathcal{J}_{n}\left(\boldsymbol{v}_{r}\right) s_{n, N_{T}}\right]
\end{gathered}
$$

where $s_{n, m}$ is the complex scattering gain between the $m$ th mode of the transmit region and $n$-th mode of the receive region, which is given by (4). Now the $\left(m, m^{\prime}\right)$-th element of $\boldsymbol{R}_{\widetilde{\boldsymbol{H}}}$, which is the correlation between $m$-th and $m^{\prime}$-th modes at the transmit region due to the $r$-th receiver, is given by

$$
\begin{aligned}
\left\{\boldsymbol{R}_{\widetilde{H}}\right\}_{m, m^{\prime}} & =E\left\{\sum_{n=-N_{R}}^{N_{R}} \sum_{n^{\prime}=-N_{R}}^{N_{R}} \mathcal{J}_{n}\left(\boldsymbol{v}_{r}\right) \overline{\mathcal{J}}_{n^{\prime}}\left(\boldsymbol{v}_{r}\right) s_{n, m} s_{n^{\prime}, m^{\prime}}^{*}\right\} \\
& =\sum_{n=-N_{R}}^{N_{R}} \sum_{n^{\prime}=-N_{R}}^{N_{R}} \mathcal{J}_{n}\left(\boldsymbol{v}_{r}\right) \overline{\mathcal{J}}_{n^{\prime}}\left(\boldsymbol{v}_{r}\right) \gamma_{m, m^{\prime}}^{n, n^{\prime}}
\end{aligned}
$$

where

$$
\gamma_{m, m^{\prime}}^{n, n^{\prime}}=E\left\{s_{n, m} s_{n^{\prime}, m^{\prime}}^{*}\right\}
$$

Substituting (4) in (8) gives

$$
\begin{aligned}
\gamma_{m, m^{\prime}}^{n, n^{\prime}} & =\int_{4} E\left\{g(\phi, \varphi) g^{*}\left(\phi^{\prime}, \varphi^{\prime}\right)\right\} e^{i\left(m-N_{T}-1\right) \phi} e^{-i\left(m^{\prime}-N_{T}-1\right) \phi^{\prime}} \\
& \times e^{-i\left(n-N_{R}-1\right) \varphi} e^{i\left(n^{\prime}-N_{R}-1\right) \varphi^{\prime}} \mathrm{d} \phi \mathrm{d} \varphi \mathrm{d} \phi^{\prime} \mathrm{d} \varphi^{\prime},
\end{aligned}
$$

where we have introduced the shorthand $\int_{4} \triangleq \iint_{\mathbb{S}^{1} \times \mathbb{S}^{1}} \iint_{\mathbb{S}^{1} \times \mathbb{S}^{1}}$.

Assume that the scattering from one direction is independent of that from another direction for both the receiver and the transmitter apertures, then the second-order statistics of the scattering gain function $g(\phi, \varphi)$ can be written as

$$
E\left\{g(\phi, \varphi) g^{*}\left(\phi^{\prime}, \varphi^{\prime}\right)\right\}=G(\phi, \varphi) \delta\left(\phi-\phi^{\prime}\right) \delta\left(\varphi-\varphi^{\prime}\right),
$$

where $G(\phi, \varphi)=E\left\{|g(\phi, \varphi)|^{2}\right\}$ with normalization $\iint_{\mathbb{S}^{1} \times \mathbb{S}^{1}} G(\phi, \varphi) d \varphi d \phi=1$. As a result, (9) simplifies to

$$
\gamma_{m, m^{\prime}}^{n, n^{\prime}}=\iint_{\mathbb{S}^{1} \times \mathbb{S}^{1}} G(\phi, \varphi) e^{i\left(m-m^{\prime}\right) \phi} e^{-i\left(n-n^{\prime}\right) \varphi} \mathrm{d} \phi \mathrm{d} \varphi .
$$

Note that $G(\phi, \varphi)$ is the normalized joint azimuth power distribution of the scatterers surrounding the transmitter and receiver regions. When the azimuth power distribution (APD) at the transmitter is independent of APD at the receiver, i.e.,

$$
G(\phi, \varphi)=\mathcal{P}_{T x}(\phi) \mathcal{P}_{R x}(\varphi),
$$

we can write the correlation between two distinct modal pairs as the product of corresponding modal correlations at the transmitter and the receiver

$$
\gamma_{m, m^{\prime}}^{n, n^{\prime}}=\gamma_{m, m^{\prime}} \gamma^{n, n^{\prime}}
$$

Condition (12) also yields that:

- modal correlation at the transmitter $\gamma_{m, m^{\prime}}$ is independent of the mode selected from the receiver region and

- modal correlation at the receiver $\gamma^{n, n^{\prime}}$ is independent of the mode selected from the transmitter region.

Therefore, from (11), the correlation between the $m$-th and $m^{\prime}$-th modes at the transmitter region due to any mode at the receiver region is given by

$$
\gamma_{m, m^{\prime}}=\int_{\mathbb{S}^{1}} \mathcal{P}_{T x}(\phi) e^{i\left(m-m^{\prime}\right) \phi} d \phi,
$$

where $\mathcal{P}_{T x}(\phi)=\int_{\mathbb{S}^{1}} G(\phi, \varphi) d \varphi$ is the normalized APD at the transmitter region. Similarly, the correlation between the $n$-th and $n^{\prime}$-th modes at the receiver region due to any mode at the transmitter region is given by

$$
\gamma^{n, n^{\prime}}=\int_{\mathbb{S}^{1}} \mathcal{P}_{R x}(\varphi) e^{-i\left(n-n^{\prime}\right) \varphi} d \varphi
$$

where $\mathcal{P}_{R x}(\varphi)=\int_{\mathbb{S}^{1}} G(\phi, \varphi) d \phi$ is the normalized APD of the scatterers surrounding the receiver antenna region.

In the current problem, we assumed that modes at the receiver region are uncorrelated, i.e., $\gamma^{n, n^{\prime}}=0$ for $n \neq n^{\prime}$ and $\gamma^{n, n^{\prime}}=1$ for $n=n^{\prime}$. Thus, applying (13) on (7) gives the correlation between $m$-th and $m^{\prime}$-th modes at the transmit region due to the $r$-th receiver as

$$
\left\{\boldsymbol{R}_{\widetilde{\boldsymbol{H}}}\right\}_{m, m^{\prime}}=\gamma_{m, m^{\prime}} \sum_{n=-N_{R}}^{N_{R}}\left|\mathcal{J}_{n}\left(\boldsymbol{v}_{r}\right)\right|^{2} .
$$


From Gegenbauer's Addition Theorem [15, page 363] we have $\lim _{N_{R} \rightarrow \infty} \sum_{n=-N_{R}}^{N_{R}}\left|\mathcal{J}_{n}\left(\boldsymbol{v}_{r}\right)\right|^{2}=1$. Then $\left\{\boldsymbol{R}_{\widetilde{\boldsymbol{H}}}\right\}_{m, m^{\prime}}$ simplifies to

$$
\left\{\boldsymbol{R}_{\widetilde{\boldsymbol{H}}}\right\}_{m, m^{\prime}}=\int_{\mathbb{S}^{1}} \mathcal{P}_{T x}(\phi) e^{i\left(m-m^{\prime}\right) \phi} d \phi .
$$

Eq. (17) suggests that when the modes at the receiver region are uncorrelated, the correlation between different modes at the transmitter is independent of the receiver antenna selected from the receiver array. Note that, $\mathcal{P}_{T x}(\phi)$ can be modeled using all common azimuth power distributions such as Uniform, Laplacian, Gaussian, von-Mises, etc.

It was shown in [16] that all azimuth power distribution models give very similar correlation values for a given angular spread, especially for small antenna separations. Therefore, without loss of generality, we restrict our investigation only to the Uniform limited azimuth power distribution, which is defined as follows.

Uniform-limited azimuth power distribution: When the energy is departing uniformly to a restricted range of azimuth angles $\pm \triangle$ around a mean angle of departure (AOD) $\phi_{0} \in[-\pi, \pi)$, we have the uniform limited azimuth power distribution [17]

$$
\mathcal{P}(\phi)=\frac{1}{2 \triangle}, \quad\left|\phi-\phi_{0}\right| \leq \triangle,
$$

where $\triangle$ represents the non-isotropic parameter of the azimuth power distribution, which is related to the standard deviation of the distribution (angular spread $\sigma=\triangle / \sqrt{3}$ ). For the above APD, the $\left(m, m^{\prime}\right)$-th entry of $\boldsymbol{R}_{\widetilde{\boldsymbol{H}}}$ is given by

$$
\begin{aligned}
\left\{\boldsymbol{R}_{\widetilde{\boldsymbol{H}}}\right\}_{m, m^{\prime}} & =\gamma_{m, m^{\prime}}=\gamma_{m-m^{\prime}} \\
& =\operatorname{sinc}\left(\left(m-m^{\prime}\right) \triangle\right) e^{i\left(m-m^{\prime}\right) \phi_{0}} .
\end{aligned}
$$

Figure 1 shows the modal correlation at the transmitter region for a uniform limited azimuth power distribution with mean AOD $\phi_{0}=0^{\circ}$. From Figure 1 it is observed that the modal correlation decreases as the non-isotropic parameter increases. Also we observe a rapid reduction of modal correlation for well separated mode orders, e.g. for large $m-m^{\prime}$. More importantly, we can observe that adjacent modes contribute to higher correlation than well separated mode orders, e.g. $\gamma_{1}$ for $m-m^{\prime}=1$. Therefore the goal is to eliminate the correlation between adjacent modes by allocating zero power to every second effective mode at the transmitter region.

\section{Optimum Power LoAding Scheme}

Writing $\boldsymbol{J}_{T}$ as the singular value decomposition (svd) $\boldsymbol{J}_{T}=$ $\boldsymbol{U}_{T} \boldsymbol{\Lambda}_{T} \boldsymbol{V}_{T}^{\dagger}$, then the capacity upper bound (6b) becomes

$$
C=\log \left|\boldsymbol{I}_{n_{T}}+\boldsymbol{U}_{T}^{\dagger} \boldsymbol{Q} \boldsymbol{U}_{T} \boldsymbol{T}\right|,
$$

where $\boldsymbol{T}=\boldsymbol{\Lambda}_{T} \boldsymbol{\Lambda}_{T}^{\dagger}$ is a diagonal matrix with squared singular values of $\boldsymbol{J}_{T}$ (or the eigen-values of spatial correlation matrix $\boldsymbol{J}_{T} \boldsymbol{J}_{T}^{\dagger}$ ) on the diagonal. Using the channel decomposition (2), the received signal at the $2 M_{\mathrm{T}}+1$ effective transmit modes

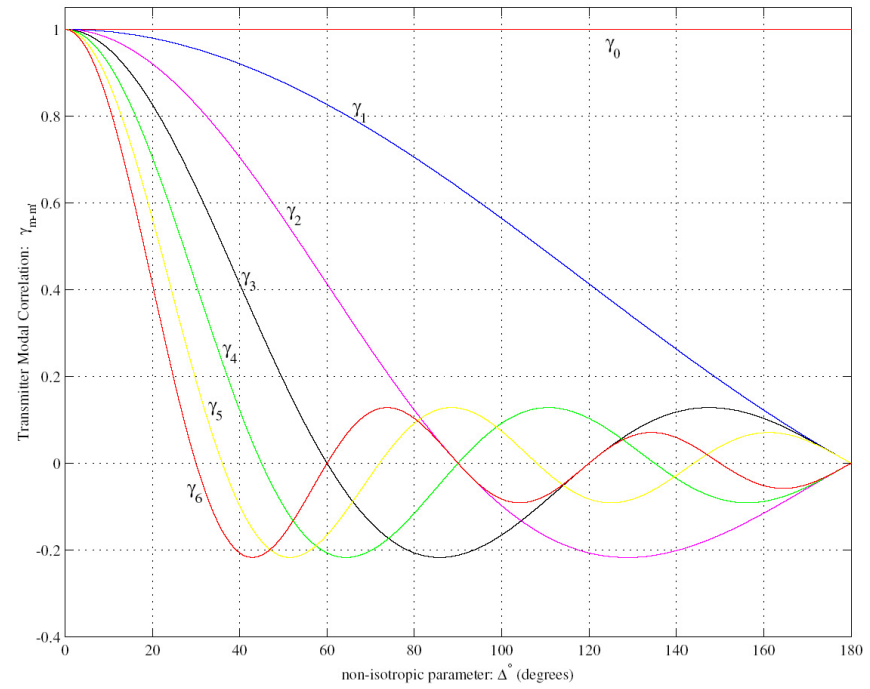

Fig. 1. Modal correlation vs non-isotropic parameter $\Delta$ of a uniform limited azimuth power distribution at the transmitter region for a mean $\operatorname{AOD} \phi_{0}=0^{\circ}$.

in the scatter-free transmit region can be written as $\boldsymbol{z}=\boldsymbol{J}_{\mathrm{T}}^{\dagger} \boldsymbol{x}$. The covariance matrix of $z$ is then given by

$$
\begin{aligned}
\boldsymbol{M}_{\mathrm{P}} & =E\left\{\boldsymbol{z} \boldsymbol{z}^{\dagger}\right\},=\boldsymbol{J}_{\mathrm{T}}^{\dagger} \boldsymbol{Q} \boldsymbol{J}_{\mathrm{T}}, \\
& =\boldsymbol{V}_{\mathrm{T}} \boldsymbol{\Lambda}_{\mathrm{T}}^{\dagger} \boldsymbol{U}_{\mathrm{T}}^{\dagger} \boldsymbol{Q} \boldsymbol{U}_{\mathrm{T}} \boldsymbol{\Lambda}_{\mathrm{T}} \boldsymbol{V}_{\mathrm{T}}^{\dagger} .
\end{aligned}
$$

Note that the $(m, m)$-th diagonal element of $\boldsymbol{M}_{\mathrm{P}}$ gives the average power allocated to the $m$-th mode in the transmitter region.

The optimum input signal covariance $\boldsymbol{Q}$ is obtained by maximizing the capacity upper bound (20) subject to transmit power constraints. The optimization problem in this case can be written as follows:

$$
\max \log \left|\boldsymbol{I}_{n_{T}}+\boldsymbol{U}_{T}^{\dagger} \boldsymbol{Q} \boldsymbol{U}_{T} \boldsymbol{T}\right|
$$

subject to $\boldsymbol{Q} \succeq 0, \operatorname{tr}\{\boldsymbol{Q}\}=P_{T}, \operatorname{tr}\left\{\boldsymbol{U}_{T}^{\dagger} \boldsymbol{Q} \boldsymbol{U}_{T} \boldsymbol{T}\right\}=P_{T}$,

$$
\left[\boldsymbol{V}_{\mathrm{T}} \boldsymbol{\Lambda}_{\mathrm{T}}^{\dagger} \boldsymbol{U}_{\mathrm{T}}^{\dagger} \boldsymbol{Q} \boldsymbol{U}_{\mathrm{T}} \boldsymbol{\Lambda}_{\mathrm{T}} \boldsymbol{V}_{\mathrm{T}}^{\dagger}\right]_{m, m}=0,
$$

where $m \in\left[1, \cdots, 2 M_{\mathrm{T}}+1\right]$ is the transmit mode (or modes) subject to power nulling and we assumed $Q$ is non-negative definite $(\boldsymbol{Q} \succeq \mathbf{0})$. Note that power constraint $\operatorname{tr}\{\boldsymbol{Q}\}=P_{T}$ ensures the total power transmitted from $n_{T}$ transmit antennas is $P_{T}$ and the second power constraint $\operatorname{tr}\left\{\boldsymbol{U}_{T}^{\dagger} \boldsymbol{Q} \boldsymbol{U}_{T} \boldsymbol{T}\right\}=P_{T}$ ensures the total power assigned to effective modes at the scatter-free transmit region is also $P_{T}$.

Let $\widetilde{\boldsymbol{Q}}=\boldsymbol{U}_{T}^{\dagger} \boldsymbol{Q} \boldsymbol{U}_{T}$. Since $\boldsymbol{U}_{T}$ is unitary, maximization/minimization over $Q$ can be carried equally well over $\widetilde{Q}$. Furthermore, $\widetilde{Q}$ is non-negative definite since $Q$ is nonnegative definite. Therefore, the optimization problem (21) becomes ${ }^{4}$

$$
\begin{array}{cc}
\min & -\log \left|\boldsymbol{I}_{n_{T}}+\widetilde{\boldsymbol{Q}} \boldsymbol{T}\right| \\
\text { subject to } & \widetilde{\boldsymbol{Q}} \succeq 0, \operatorname{tr}\{\widetilde{\boldsymbol{Q}}\}=P_{\mathrm{T}}, \operatorname{tr}\{\widetilde{\boldsymbol{Q}} \boldsymbol{T}\}=P_{\mathrm{T}}, \\
& {\left[\boldsymbol{V}_{\mathrm{T}} \boldsymbol{\Lambda}_{\mathrm{T}}^{\dagger} \widetilde{\boldsymbol{Q}} \boldsymbol{\Lambda}_{\mathrm{T}} \boldsymbol{V}_{\mathrm{T}}^{\dagger}\right]_{m, m}=0 .}
\end{array}
$$

\footnotetext{
${ }^{4}$ Maximization of $f(x)$ is equivalent to minimization of $-f(x)$.
} 


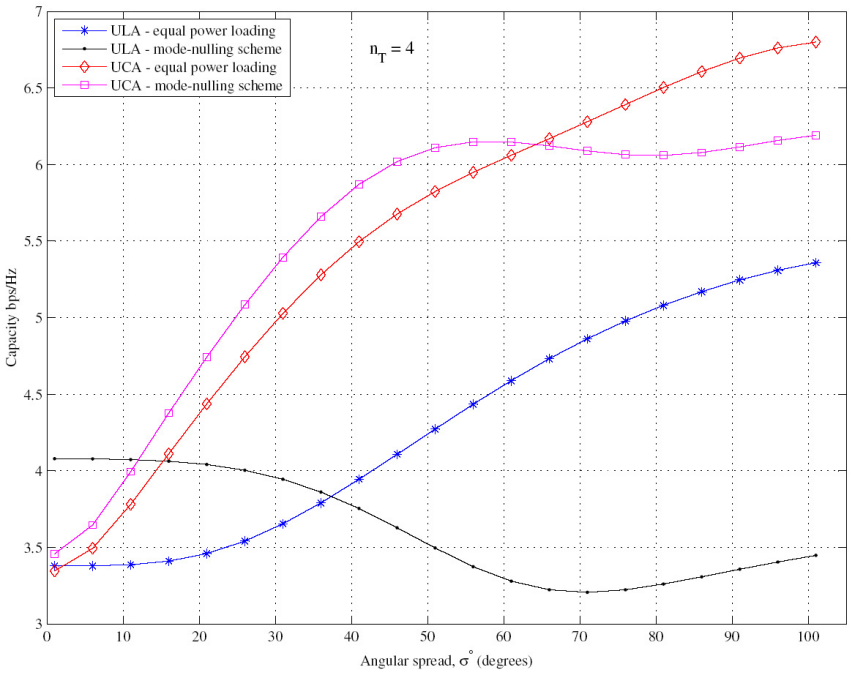

Fig. 2. Capacity comparison between power-loading schemes for a uniform limited azimuth power distribution at the transmitter with mean AOD $\phi_{0}=0^{\circ}$ for increasing angular spread: $n_{T}=4$ transmit antennas.

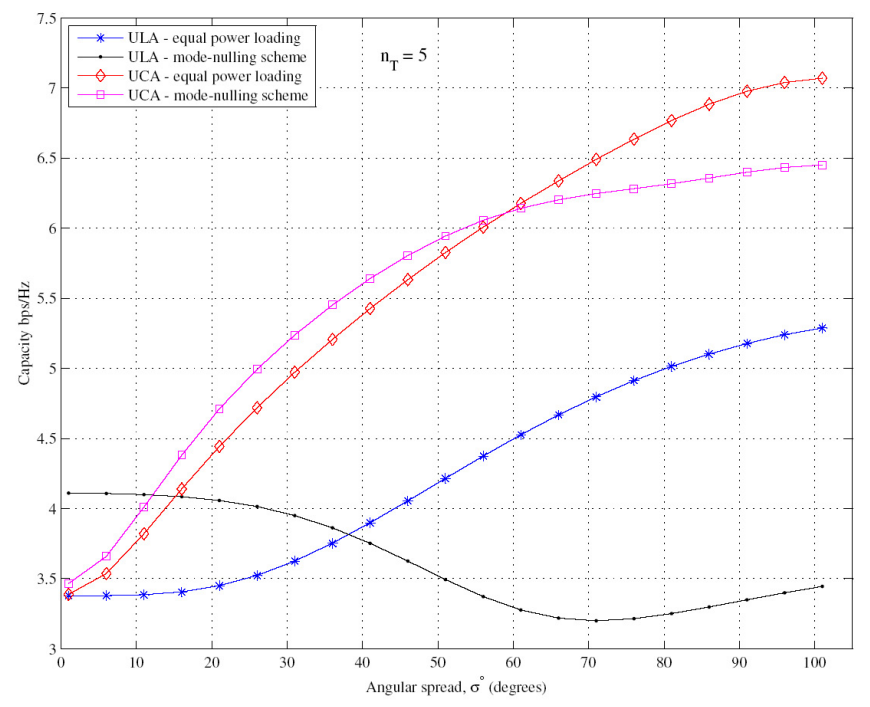

Fig. 3. Capacity comparison between power-loading schemes for a uniform limited azimuth power distribution at the transmitter with mean AOD $\phi_{0}=0^{\circ}$ for increasing angular spread: $n_{T}=5$ transmit antennas.

A closed form solution to this optimization problem is unknown. However, we can find the solution for $\widetilde{Q}$ by numerical methods such as Sequential Quadratic Programming [18].

Note that we used the capacity upper bound expression (6b) in the optimization problem (21) instead of the capacity expression (6a). As a consequence, we expect some capacity loss from new mode-nulling scheme compared to the equal power loading scheme at higher angular spread values. Note also that since we maximized (6b), the optimum $Q$ is fixed for fixed antenna placement and the transmitter does not require any form of feedback of CSI from the receiver and the optimization can be implemented off-line.

\section{NumericAl Results}

We now illustrate the capacity benefits obtained by applying the mode-nulling scheme on spatially constrained antenna arrays. We consider a MIMO system with $n_{T}=\{4,5\}$ transmit antennas constrained within a scatter-free circular region of radius $r_{\mathrm{T}}=0.25 \lambda$, corresponding to 7 effective modes at the transmitter region, and a large number of uncorrelated receive antennas for a total power budget of $P_{\mathrm{T}}=10 \mathrm{~dB}$. The transmit antennas are placed in Uniform Circular Array (UCA) and Uniform Linear Array (ULA) configurations. Figures 2 and 3 depict the capacity comparison between equal power loading (i.e., $\left.\boldsymbol{Q}=\left(P_{T} / n_{T}\right) \boldsymbol{I}_{n_{T}}\right)$ and new mode-nulling scheme for a uniform limited azimuth power distribution at the transmitter with mean AOD $\phi_{0}=0^{\circ}$ for 4 and 5 transmit antennas, respectively. For the mode-nulling scheme, we set the 4-th element of the diagonal of $\boldsymbol{V}_{\mathrm{T}} \boldsymbol{\Lambda}_{\mathrm{T}}^{\dagger} \widetilde{\boldsymbol{Q}} \boldsymbol{\Lambda}_{\mathrm{T}} \boldsymbol{V}_{\mathrm{T}}^{\dagger}$ in (22) to zero, i.e., allocate zero power to the 0 -th mode at the transmitter region.

From Figures 2 and 3 it is observed that mode-nulling scheme provides significant capacity improvements at small angular spread values, in particularly for the ULA transmit antenna configuration. However, in comparison with the capacity performance of equal power loading scheme, we observe a capacity loss from mode-nulling scheme at high angular spread values for both antenna configurations. For example, mode-nulling scheme gives poor capacity performance when $\sigma>55^{\circ}$ for UCA with 4-transmit antennas and $\sigma>30^{\circ}$ for ULA with 4-transmit antennas. To further investigate this capacity loss, we now consider the power assignment to each mode at the transmitter region by two schemes.

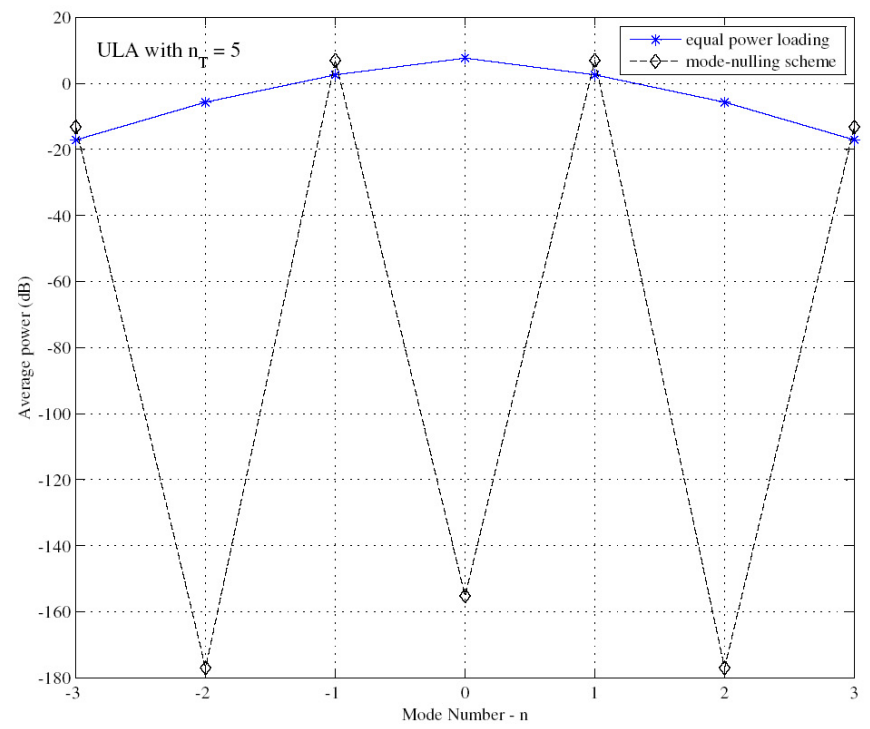

Fig. 4. Average power allocated to each effective transmit mode in a circular aperture of radius $0.25 \lambda . P_{\mathrm{T}}=10 \mathrm{~dB}$ : ULA antenna configuration, $n_{T}=5$.

Figures 4 and 5 show the average power assigned to the first 7 effective transmit modes for the case of $n_{T}=5$ for ULA and UCA transmit antenna configurations, respectively. 


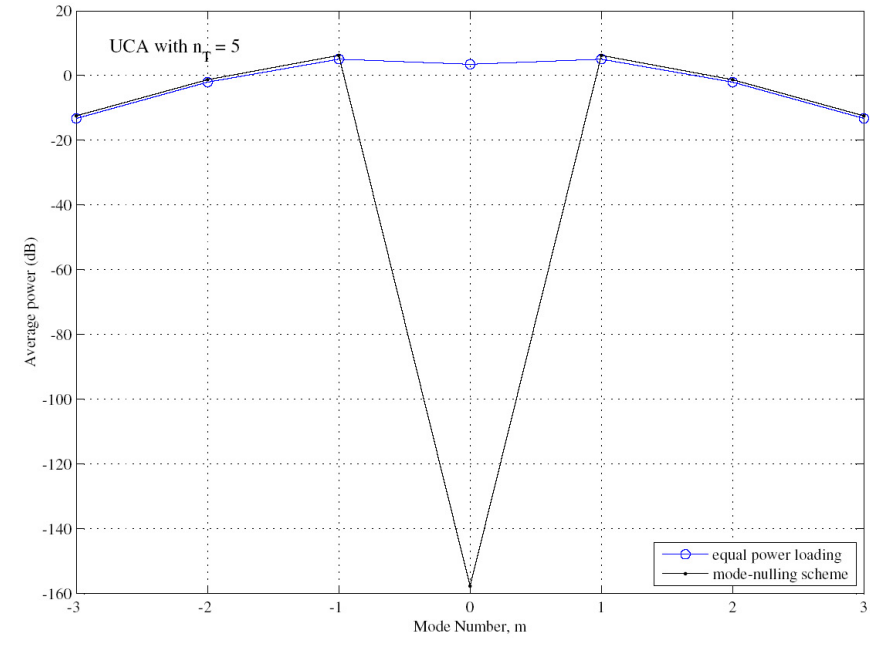

Fig. 5. Average power allocated to each effective transmit mode in a circular aperture of radius $0.25 \lambda$. $P_{\mathrm{T}}=10 \mathrm{~dB}$ : UCA antenna configuration, $n_{T}=5$.

It is observed that with the ULA antenna configuration modenulling scheme allocates considerable amount of power on to the transmit mode set $\{-3,-1,1,3\}$ and almost zero power to the rest of the available transmit modes. However, in the case of UCA, mode-nulling scheme allocates considerable amount of power on to the transmit mode set $\{-3,-2,-1,1,2,3\}$ and almost zero power to the 0 -th mode. Therefore, at high angular spread values, mode-nulling scheme does not utilize the full set of uncorrelated (or near uncorrelated) modes available at the region for transmission. As a result, mode-nulling scheme gives poor capacity performance at high angular spread values. However, capacity loss for UCA is less compared to the ULA at high angular spread values. This is because, as indicated above, the mode-nulling scheme utilizes a larger transmit mode set in the case of UCA than in the case of ULA. It is further observed that for 1-D arrays (ULA) mode-nulling scheme gives more scope for capacity improvements at low angular spread values than for 2-D arrays (UCA).

\section{CONCLUSIONS}

A new power loading scheme is proposed for improving the capacity of spatially constrained MIMO systems when the angular spread at the transmitter side is small. It was shown that the proposed new scheme provides significant capacity improvements than the traditional equal power loading scheme at low angular spread values. It was further shown that this new power loading scheme gives more scope for capacity improvements for 1-D arrays at low angular spread values than for 2-D arrays. At high angular spread values, the proposed scheme experiences some capacity loss compared to the equal power loading scheme for both types of antenna settings due to not utilizing full set of uncorrelated (or near uncorrelated) modes available at the transmitter. Since the design parameters of the power loading scheme is based on readily available antenna configuration details (antenna spacing and placement), the power loading scheme is fixed and transmitter does not require any feedback of channel state information from the receiver. This is an added advantage over the other power loading schemes found in the literature.

\section{ACKNOWLEDGEMENTS}

This work was supported by the Australian Research Council Discovery Grant DP0343804.

\section{REFERENCES}

[1] I. E. Telatar, "Capacity of multi-antenna gaussian channels," Tech. Repo., AT\&T Bell Labs, 1995.

[2] Da-Shan Shiu, G. J. Foschini, M. J. Gans, and J. M. Kahn, "Fading correlation and its effect on the capacity of multielement antenna systems," IEEE Trans. Commun., vol. 48, no. 3, pp. 502-513, 2000.

[3] S. Wei, D. Goeckel, and R. Janaswamy, "On the capacity of fixed length antenna arrays under bandlimited correlated fading," in Conf. on Inf. Sciences and Systems, CISS'02, Princeton, 2002, pp. 1088-1093.

[4] D. Gesbert, T. Ekman, and N. Christophersen, "Capacity limits of dense palm-sized MIMO arrays," in Proc. IEEE Global Telecommunications Conference, Globecom'02, Taipei, Taiwan, 2002.

[5] L. Hanlen and M. Fu, "MIMO wireless communication systems: Capacity limits for sparse scattering," in Proc. 3rd Australian Communcations Theory Workshop, AusCTW'03, Canberra, Australia, 2002.

[6] A. Goldsmith, S. A. Jafar, N. Jindal, and S. Vishwanath, "Capacity limits of MIMO channels," IEEE J. Select. Areas Commun., vol. 21, no. 5, pp. 684-702, June 2003.

[7] L. Hanlen and A. Grant, "On capacity of ergodic multiple-input multiple-output channels," in Proc. 6th Australian Communcations Theory Workshop, AusCTW'05, Brisbane, Australia, Feb. 2005, pp. 121124.

[8] A. J. Goldsmith and P. P. Varaiya, "Capacity of fading channels with channel side information," IEEE Trans. Inform. Theory, vol. 43, no. 6, pp. 1986-1992, Nov. 1997.

[9] J. Liu, N. Elia, and S. Tatikonda, "Capacity-achieving feedback scheme for flat fading channels with channel state information," in Proceedings of the 2004 American Control Conference, Boston, Massachusetts, USA, June 30 - July 2 2004, vol. 4, pp. 3593-3598.

[10] T. D. Abhayapala, T. S. Pollock, and R. A. Kennedy, "Spatial decomposition of MIMO wireless channels," in Proc. 7th International Symposium on Signal Processing and its Applications, Paris, France, July 2003, vol. 1, pp. 309-312.

[11] N. Chiurtu, B. Rimoldi, and I. E. Telatar, "Dense multiple antenna systems," in Proc. IEEE Information Theory Workshop, ITW'03, Cairns, Australia, 2001.

[12] T. D. Abhayapala, T. S. Pollock, and R. A. Kennedy, "Characterization of 3D spatial wireless channels," in Proc. IEEE Vehicular Technology Conference, VTC'03-Fall, Orlando, Florida, USA, Oct. 2003, vol. 1, pp. 123-127.

[13] H. M. Jones, R. A. Kennedy, and T. D. Abhayapala, "On dimensionality of multipath fields: Spatial extent and richness," in Proc. IEEE Int. Conf. Acoust., Speech, Signal Processing, ICASSP'02, Orlando, Florida, May 2002, vol. 3, pp. 2837-2840.

[14] T. S. Pollock, T. D. Abhayapala, and R. A. Kennedy, "Fundamental limits of MIMO capacity for spatially constrained arrays," in Proc. 4th Australian Communications Theory Workshop, AusCTW'04, Melbourne, Australia, February 2003, pp. 7-12.

[15] M. Abramowitz and I. A. Stegun, Handbook of Mathematical Functions, Dover Publications, Inc., New York, 1972.

[16] T. S. Pollock, T. D. Abhayapala, and R. A. Kennedy, "Introducing space into MIMO capacity calculations," Journal on Telecommunications Systems, Kluwer Academic Publishers, vol. 24, no. 2, pp. 415-436, 2003.

[17] J. Salz and J. H. Winters, "Effect of fading correlation on adaptive arrays in digital mobile radio," IEEE Trans. Veh. Technol., vol. 42, no. 4, pp. 1049-1057, Nov. 1994.

[18] P. E. Gill, W. Murray, and M. H. Wright, Practical Optimization, Academic Press, 1981. 